\section{Subjectivity and flow cytometric variability}

\section{Gisela Pachón, Isabel Caragol and Jordi Petriz}

We read the Review article by Holden T. Maecker and colleagues (Standardizing immunophenotyping for the Human Immunology Project. Nature Rev. Immunol. 12, 191-200 (2012) $)^{1}$ with great interest. Over the years, scientists have built up expertise in different research fields through independent coordination. Continuous quality improvement is expected in many technical and scientific settings, which will require continued coordination to prevent the duplication of resources by the national and international organizations involved.

In 2008, the International Society for Analytical Cytology (ISAC) and other interested parties introduced a new recommendation for the recording and reporting of information about the experimental details of flow cytometry experiments, including samples, instrumentation and data analysis. This recommendation is known as the Minimum Information about a Flow Cytometry Experiment (MIFlowCyt) ${ }^{2}$, and it has been developed in a coordinated manner with checklists for other high-throughput experiments; together, these recommendations constitute the Minimum Information for Biological and Biomedical Investigations (MIBBI) project ${ }^{3}$. Moreover, the United Kingdom National External Quality Assessment Service (UK NEQAS), the EuroFlow Consortium and the Subcommittee on Quality Assessment of Haematopoietic Stem Cell Grafts (established by the European Group for Bone Marrow Transplantation) facilitate a comprehensive external quality assessment service and provide guidelines on laboratory medicine for optimal patient care. Through education and the promotion of best practice, different supra-national organizations are working to improve the comparability and the reliability of the results obtained from flow cytometry (and other) experiments, wherever these are carried out. Although flow cytometry is mostly carried out in a research setting, the above-mentioned framework is also contributing to a decrease in variability in clinical studies.

Advanced polychromatic flow cytometry (involving 16 or more fluorophores) has added more complexity to immunophenotyping studies, and we believe that Fluorescence Minus One (FMO) controls ${ }^{4}$ are the best choice to accurately identify target cells in this setting. However, are all laboratories economically and technically able to include FMO controls for immunophenotyping standardization? And how can we persuade hospital managers about the need to use these optimal controls in clinical settings despite their greater cost?

Since 1995, the Working Groups of the Sociedad Ibérica de Citometría (SIC) have supported a multi-laboratory quality-control task force with the aim of decreasing both intra- and inter-laboratory variation of flow cytometry-based measurements. A reference laboratory coordinates a series of intercalibration studies, the results of which are actively discussed at our national meetings. The SIC Working Groups have successfully contributed to decreasing intra- and interlaboratory variation. Ongoing SIC studies focus on laboratory accreditation, mainly for the absolute counting of lymphocytes and of CD $34^{+}$cells. The experience of the SIC Working Group for counting CD $34^{+}$cells has shed more light on inter-laboratory comparison studies. After two consecutive trials, a marked variation in terms of $\mathrm{CD} 34^{+}$cell measurements was detected. To eliminate the 'noise' resulting from differences in sample manipulation, instrumentation and the reagents used, we initiated a multi-centre in silico trial to focus on the subjectivity of data analysis from flow cytometric studies. For this new trial, a reference laboratory sent four different representative flow cytometry data files to 50 laboratories for independent and blind analysis, together with specific recommendations for the analysis of those files. Specimens had been stained in the reference laboratory according to a reference protocol for the absolute counting of CD $34^{+}$cells ${ }^{5}$. Surprisingly, the results of this in silico multi-centre trial ${ }^{6}$ showed unexpectedly high coefficients of variation between laboratories, ranging from $5.52 \%$ to $13.64 \%$ for absolute counts of CD $45^{+}$ events, from $12.62 \%$ to $58.72 \%$ for CD $34^{+}$ events, and from $1.10 \%$ to $6.00 \%$ for fluorescent microspheres. These observations could have implications for both research and clinical studies, in particular when dealing with rare cell populations (such as $\mathrm{CD} 34^{+}$cells). However, the more abundant $\mathrm{CD} 45^{+}$cells also had high coefficients of variation in this study.

As a concluding remark, we believe that specific professional training should help to decrease the subjectivity-related variability that we observed. We believe that new efforts to include in silico studies in the Human Immunology Project could contribute substantially to the standardization of immunophenotyping. Further common supra-national strategies for immunophenotyping would also help to generate reference standards and 'cytomic' databases, allowing for integration with existing genomic and proteomic databases.

Gisela Pachón is at the Grup d'Investigació en Diabetis i Alteracions Metabòliques Associades, CIBERDEM, Institut d'Investigació Sanitària Pere i Virgili (IISPV), Tarragona 43007, Spain.

Isabel Caragol and Jordi Petriz are at the Vall d'Hebron Institut de Recerca (VHIR), Barcelona 08035, Spain.

Correspondence to J.P. e-mail: jordi.petriz@vhir.org doi: 10.1038/nri3158-c1

1. Maecker, H. T., McCoy, J. P. \& Nussenblatt, R. Standardizing immunophenotyping for the Human Immunology Project. Nature Rev. Immunol. 12,

191-200 (2012)

2. Lee, J. A. et al. MIFlowCyt: the minimum information about a Flow Cytometry Experiment. Cytometry A 73 926-930 (2008)

3. Taylor, C. F. et al. Promoting coherent minimum reporting guidelines for biological and biomedical investigations: the MIBBI project. Nature Biotech. 26 889-896 (2008)

4. Roederer, M. Spectral compensation for flow cytometry: visualization artifacts, limitations, and caveats. Cytometry 45, 194-205 (2001).

5. Fornas, O. et al. Flow cytometry counting of $\mathrm{CD} 34^{+}$ cells in whole blood. Nature Med. 6, 833-836 (2000).

6. Petriz, J. for the CD34 Working Group. Tercer estudio multicéntrico para el recuento de células CD34 mediante citometría de flujo. in VIII Congreso de la Sociedad Ibérica de Citometría (Book of abstracts) Oral communication 20, 108 (2003).

Competing interests statement

The authors declare no competing financial interests. 